\title{
Pipeline Sizing Based on Computer Simulation
}

$F_{1}$ ow-diversion treatment of cerebral aneurysms with the Pipeline Embolization Device (Covidien, Irvine, California) has been around for nearly 13 years, yet we are still struggling with some fundamental issues, such as optimal device-size selection and optimal flow diversion (ie, which aneurysms need $>1$ device for a cure, and how we can identify those aneurysms).

As operators, we all deal with these questions differently. Some of us use as individualized an approach to each patient and aneurysm as possible and spend considerable time ruminating over minute differences in device length and diameter to be implanted, not to mention the guesswork that goes into predicting how much flow diversion is necessary or enough to ensure complete aneurysm occlusion after the first treatment (ie, do we use 1 or multiple devices?). This desire to cure with a single procedure may be rooted in our prior experience and frustration with the predictability of recurrence after more traditional endovascular therapy of complex aneurysms. Others among us have given up the guessing and decided to bring the same approach to each aneurysm we encounter: Always use 1 device, and always use the widest and longest one ("to be safe"). We all hope that one day artificial intelligence will tell us exactly what kind, size, and quantity of devices or materials we need to deliver to optimally treat our target-be it an aneurysm, a brain AVM, and so forth.

At the same time, I think we also fear the arrival of that very same day.

The article written by Ospel et $\mathrm{al}^{1}$ brings us a bit closer to our dream (but luckily leaves enough left to think about before we get there).

The authors of the article embarked on an experiment to assess the potential use of a virtual-simulation software in planning Pipeline flow diversion in 74 aneurysms. Due to limitations of the simulation software, the simulation was applied to a single Pipeline device (though 7 cases were treated with multiple devices; in these, the software was used for the first device only). One of the 2 participating hospitals, contributing 63 aneurysms, used manual measurements based on standard angiographic evaluation (including 3D images) to select device sizes, and simulation was performed at a later date blinded to the device-size selection of the treating physician. The other hospital enrolled 11 aneurysms, all treated after the simulation software became available; therefore, they applied the software to determine optimal device size, and manual measurements were obtained at a later time blinded to the results of the simulation.

Overall, the authors found that the simulation software suggested somewhat shorter devices and this difference reached statistical significance. Intradural aneurysms and, within them, more distal aneurysms were especially likely to have a shorter device recommendation by the software, though the length difference between manual and simulation measurements was smaller in intradural aneurysms. On the other hand, they found no statistically significant difference in device diameters suggested by the software versus manual measurements. Nevertheless, not surprisingly, they observed that the largest discrepancies in sizing (both length and diameter) of the devices, as suggested by manual measurements and simulation, occurred in relation to extradural aneurysms that were large, fusiform, or dissecting.

Therefore, the authors concluded that software-based sizing may be most beneficial for intradural aneurysms, in which considerations such as the avoidance of perforators are significantly different from those at extradural sites.

An additional, not unexpected finding is the low rate of agreement in device sizing between the 2 measurement methods. The significance of this is unclear because if we consider all the various lengths and diameters, one can choose from $>90$ device sizes. It is very likely that the agreement rate on recommended optimal device sizes for a set of aneurysms between 2 experienced operators would also be very low. Therefore, it would be difficult to conclude that the software is better at predicting device sizes purely on the basis of the frequency of this discordance.

The authors should be applauded for this well-written and thoughtful article. The very fact that they are making an effort to improve Pipeline device sizing demonstrates their commitment to advancing our understanding of flow diversion. As shown in numerous articles in the past, optimal device length and diameter selection are critical in providing more appropriate deployment, wall apposition, and device porosity at the aneurysm neck and, as a result, improved flow diversion. Therefore, it logically follows that better pre- 
diction of device sizing should theoretically lead to better outcomes. This study is a small step in that direction.

Disclosures: I am a consultant and Pipeline proctor for Medtronic; I have no financial or other interest in the simulation software discussed in this article. One of the authors was, in part, trained by me; however, this was unknown to me at the time of my initial review of the article.

\section{REFERENCE}

1. Ospel JM, Gascou G, Costalat V, et al. Comparison of Pipeline embolization device sizing based on conventional 2D measure- ments and virtual simulation using the Sim\&Size software: an agreement study. AJNR Am J Neuroradiol 2019;40:524-30. Neuroendovascular Surgeon UNC Rex Hospital Raleigh, NC Adjunct Associate Professor of Neurology University of North Carolina - Chapel Hill Chapel Hill, NC

http://dx.doi.org/10.3174/ajnr.A5998 